\title{
Improving Childhood Obesity Treatment Using New Technologies: The ETIOBE System
}

\author{
Rosa. M. Baños ${ }^{*}, 1$, Ausias Cebolla ${ }^{2,4}$, Cristina Botella ${ }^{2,4}$, Azucena García-Palacios ${ }^{2,4}$, Elia Oliver ${ }^{1}$, \\ Irene Zaragoza ${ }^{3,4}$ and Mariano Alcaniz \\ ${ }^{1}$ Universidad de Valencia, Spain \\ ${ }^{2}$ Universitat Jaume I de Castellón, Spain \\ ${ }^{3}$ Instituto de Investigación en Bioingenieria y Tecnología Centrad en el Ser Humano. I3BH. Universidad Politécnica de \\ Valencia, Spain \\ ${ }^{4}$ CIBER Fisiopatología Obesidad y Nutrición (CB06/03), Instituto Carlos III, Spain
}

\begin{abstract}
Childhood obesity is an increasing public health problem in western culture. Sedentary lifestyles and an "obesogenic environment" are the main influences on children leading to an increase in obesity. The objective of this paper is to describe an e-health platform for the treatment and prevention of childhood obesity called ETIOBE. This e-health platform is an e-therapy system for the treatment of obesity, aimed at improving treatment adherence and promoting the mechanisms of self-control in patients, to obtain weight loss maintenance and to prevent relapse by establishing healthy lifestyle habits. ETIOBE is composed of three different applications, the Clinician Support System (CSS), the Home Support System (HSS) and the Mobile Support System (MSS). The use of new Information and Communication (ICT) technologies can help clinicians to improve the effectiveness of weight loss treatments, especially in the case of children, and to achieve designated treatment goals.
\end{abstract}

Keywords: Obesity, e-health, e-therapy, persuasive technology, cognitive-behavioural treatment.

Obesity is a chronic disease that is reaching alarming rates associated with huge economic, social and personal costs, not only because of its direct consequences, but also because of the enormous additional risk of developing a range of life-threatening illnesses and psychological problems. According to the European Association for the Study of Obesity [1], although there are powerful genetic factors which heighten the risk of developing obesity, the causes of this epidemic are mainly twofold: a) an increase the abundance of "energy dense" food and drink, which promote excessive "calorie" consumption and support a ubiquitous "snacking" culture, and b) the systematic public and commercial developments that restrict opportunities for physical activity. New approaches are needed to address the challenge of preventing and treating obesity, particularly in the younger generation. Childhood obesity, especially in the teen years, is a powerful predictor of obesity in adults [2], and as the most common childhood disorder in Europe it is an increasingly common public health problem [1].

The treatment of childhood obesity should be multidisciplinary, involving both medical and psychological input. Guidelines for psychological interventions, (e.g. National Institute for Health and Clinical Excellence) [3,4] recommend cognitive-behavioral therapy (CBT) as the treatment of choice [5] due to its focus on strategies which aim to in

*Address correspondence to this author at the Departamento de Personalidad, Evaluación y Tratamientos Psicológicos, Facultad de Psicología Avda. Blasco Ibáñez, 2146010 - Valencia. Spain; Tel: 34- 963- 864412;

E-mail:banos@uv.es crease activity levels and improve eating habits. CBT obesity programs propose that instead of putting pressure on people to follow a life-long diet (which evidence to date suggests is an unrealistic an unsustainable therapeutic objective) it is more important to promote change in eating and activitybased habits and to learn to balance energy consumption and expenditure. With this aim, several treatment components are included to promote changes in behaviour, cognitions and emotional patterns that contribute to obesity [6].

The National Heart, Lung, and Blood Institute [7] advise that obesity treatment programmes should aim to reduce weight by $10 \%$, and to maintain $5 \%$ of weight loss in the following 6 months. But research outcome data has not been so optimistic. Unfortunately, patients tend to recover between $30 \%$ and $35 \%$ of the weight they had lost in the first year following treatment, and some studies suggest that $100 \%$ of the weight that has been lost is regained after 5 years [8]. Elfhag and Rössner [9] completed a detailed review of obesity research outcome studies and concluded that intrinsic motivation to change is the best predictor of weight loss maintenance, because of the effort required to adhere to the programme over the long-term. Another strong predictor of weight reduction is adherence to daily homework tasks and activities prescribed [10]. The success of the treatment depends on the degree to which patients can make changes to lifestyles habits, and continue to follow therapeutic advice. If patients do not receive continuous support, they are faced with numerous obstacles to recovery and increased risk of treatment failure. 
The new Information and Communication (ICT) technology can help to achieve this goal, by offering constant support, increasing motivation to change, enhancing problem insight and contingently rewarding desirable behaviours, in order to strengthen users' resolve to make changes in the face of an environment which does not favour healthy lifestyle habits. ICTs (Internet, mobile phones, Personal Digital Assistants (PDAs), etc.) have several advantages, such as providing immediate information to users, reminding them of daily tasks (physical exercise, diet, etc.) and giving guidance and advice (e.g., how to control snacking, how to manage urges to eat, etc.). Clinicians cannot give this immediate information to each patient every day, but ICT can. Therefore, ICT may be a very useful way of increasing obesity treatment efficacy.

The objective of this paper is to describe the ETIOBE platform, an e-health therapeutic system developed to help the clinicians working in childhood weight loss treatment programmes.

\section{The ETIOBE Platform}

The ETIOBE platform is an e-therapy system for the treatment of obesity, aimed at improving treatment adherence, promoting the mechanisms of patient self-control facilitating the maintenance of weight loss and preventing relapses by means of the establishment of healthy lifestyle habits.

ETIOBE is supported by different ICTs able to transfer, manage, store, interpret and react to information; and also to customize and adapt treatment strategies according to individual patient characteristics and responses.

The ETIOBE platform is supported by sensors, that enable the clinician to obtain relevant information from patients (contextual, physiological and psychological), as well as a range of communication and informatics applications capable of reacting to such information, which is flexible enough to react appropriately according to the personal responses and circumstances of individual patients..

ETIOBE is composed of three applications: Clinical support system (CSS), a tool where the clinician can design an intervention, and then adapt it according to how the patient changes and progresses. This platform is connected in real time with two other platforms, allowing the clinician to be informed about patients at any time. The second one is the Home support system (HSS). This is a website where children can find the tasks that their clinician has prescribed (self-reports of dietary and physical activity, etc.). A nutritional and healthy lifestyle knowledge component is also included with the support of several "educational games" that aim to facilitate the learning process. The third application is the Mobile support system (MSS). This one is composed of a self-report diary for dietary and physical activity based on PDA technology, and also a sensorization and physiological information monitor that is connected in real time to the CSS. A more detailed description of each application is provided below.

\section{Clinical Support System (CSS)}

This application is used by clinicians, who incorporate the medical and psychological data enabling them to create a patient's profile. The questionnaires are computerized and patients can input responses directly onto a computer screen. Questionnaire results are easily accessible and are incorporated immediately into each child's profile. Furthermore the system has inbuilt alarms, such that if questionnaire responses indicate that the child is at some kind of risk (physical or psychological) the system alerts the health professional.

Furthermore, this application offers the clinician the opportunity to adapt the intervention program around the individual requirements of each patient, and to monitor and make changes according to the patient's progress (Figs. 1 and 2). The therapist can monitor children's progress in real time, and reinforce treatment adherence (eating habit changes, homework tasks, physical exercise, etc.) through feedback within the other two platforms (HSS and MSS). This information enables the clinician to change or adapt the treatment to the specific characteristics of the children in each moment, enhancing motivation to change and treatment adherence.

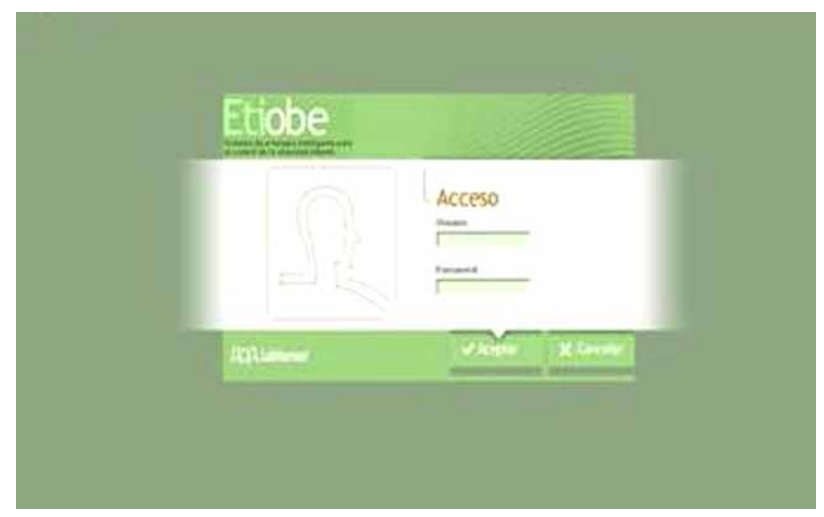

Fig. (1). Data input interface on the CSS.

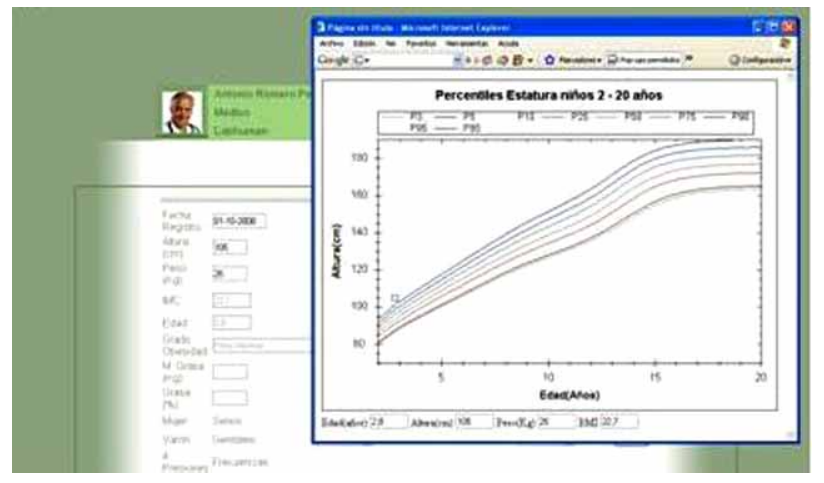

Fig. (2). Data interface on the CSS.

\section{Home Support System (HSS): The ETIOBE Mates}

This application is called "The ETIOBE mates" (Figs. 3 and 4), and it is used by patients at home, over a PC platform connected to Internet. Children can log on and contact their therapist, other users and also get access to the tasks and the activities that the clinician has programmed for them, as well as other functions (physiological measures, self-report diaries, educational games, etc.). This application also includes some amusing activities which they can participate in, such as cooking healthy recipes, or playing games. All results and data are sent to the CSS immediately. 
This application allows children to design a virtual agent or avatar, who guides them and recommends the tasks the clinician has proposed for them to carry out. This avatar also provides children with rewards. Children win points as they complete the suggested tasks, and these points allow them to pass from one level to the next (moving from 'beginners' to 'experts'). Patients know how many points that they can win by carrying out each task, playing educational games, completing self-report diaries, and so on.

The platform also includes a nutritional knowledge component, healthy food recipes, information about the benefits of physical activity and sports, and access to the user profile where they have access to all the information (task level, ranking, social network). Furthermore, this application includes several educational games. Educational games integrate learning and play, making the process of learning new concepts, skills, etc. more attractive for children, which can help to improve and facilitate the learning of this kind of information. The research literature shows that the use of computer games is an effective tool for learning psychological skills and educational learning. Different studies show that the educational games accelerate the learning process and improve motivation levels [11]. There are several advantages of an educative tool such as this, including its use of language that is age-appropriate and a mode of delivery which is both familiar and attractive to most children (i.e. internet, computer games). It offers a great deal of flexibility (led by the clinician) and adaptability (targets can be modified according to clinical objectives), making the entire learning process more fun and therefore more selfreinforcing than most programmes.

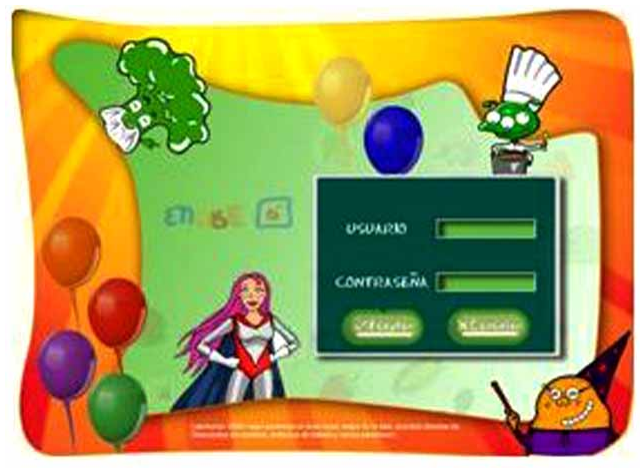

Fig. (3). HSS data entry interface.

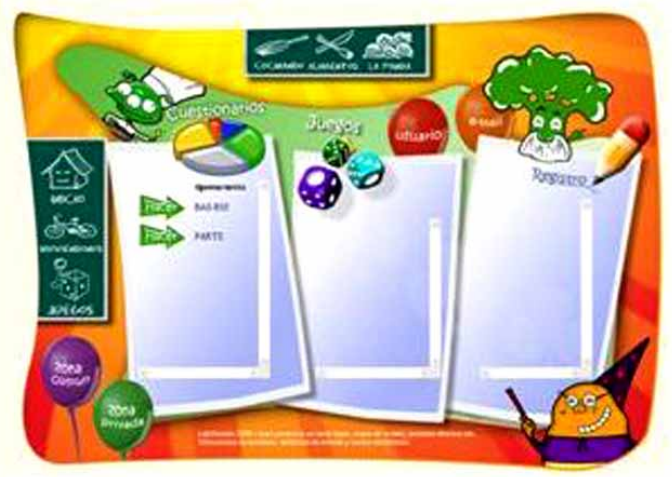

Fig. (4). HSS screen page.

Three educational games have been designed and are included: Memory, Healthy Plate, and Superetiobe.
The Memory game is aimed at helping children to learn nutritional knowledge (Fig. 5). Cards with food pictures are presented one by one. The user has to find pairs of foods in the panel. When the pair is found, the player receives points. After several questions are answered correctly, a multiplechoice nutritional quiz is presented. The user has to answer the quiz in order to keep playing and winning extra points.

The objective of Healthy Plate is to learn the categories in the food pyramid (Fig. 6). The user has to allocate several foods into the right categories and select their nutritional features. This game is designed for young children, as an introduction to the nutritional knowledge component. After each correct answer the player receives points. After several correct answers there is a multiple choice quiz similar to that in the Memory game, that should be answered correctly in order to gain extra points.

The Superetiobe game is aimed at demonstrating that some daily behaviour can be changed in an easy way, leading to a healthier life (Fig. 7). It consists of a graphic adventure, where the child has to help a detective, called Superetiobe, to solve some cases of children who have unbalanced eating habits or a sedentary lifestyle. In each game a character is presented with a range of habits that prevent a healthy lifestyle. Each case must be resolved in a limited time.

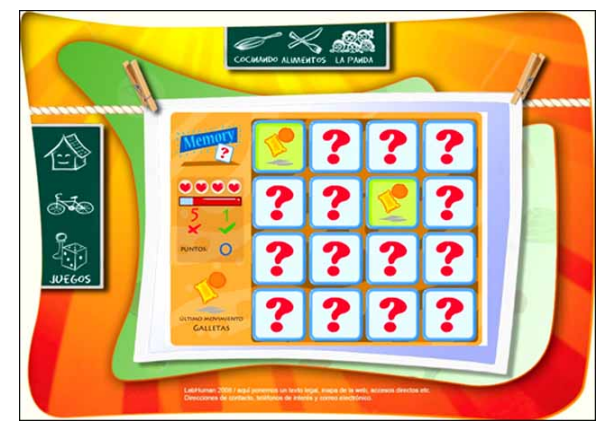

Fig. (5). The Memory Game.

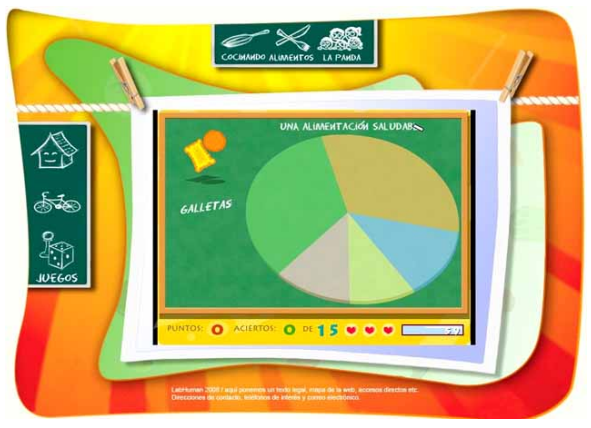

Fig. (6). The Healthy Plate game.

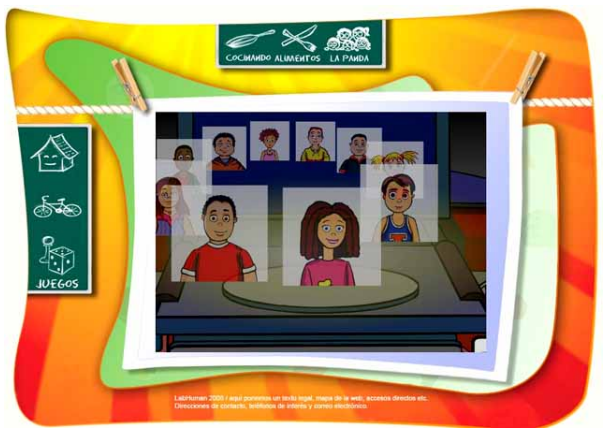

Fig. (7). The Superetiobe game. 


\section{Mobile Support System (MSS)}

The Mobile support system is composed of a Personal Digital Assistant (PDA) and a sensorization platform named TIPS (Therapy Intelligent Personal Sensor). The PDA enables the patient to establish continuous communication with the HSS application, with the CSS application, and with the personal agent or avatar. One of the most important functions of the PDA is the self-report record for recording food intake and physical activity.

Self-report techniques are considered necessary for both assessment and treatment phases. The goal is to evaluate behaviours in a natural setting (e.g. home or school). The frequency of dietary self-monitoring during weight loss treatments is a reliable predictor of success [12]. Research has indicated that the use of a PDA improves dietary selfmonitoring frequency [13-15]. Research also shows that electronic diaries improve adherence to treatments [16] and, also the levels of acceptability [17] over paper diary systems. The PDA registers the food eaten (amount, order, type), (Fig. 8), time and place where it is consumed, thoughts and emotions prior to and after eating (Fig. 9), and physical activities undertaken. For more detailed information about the PDA self-report system see Baños et al. [18].

The sensorization application inside the MSS is called TIPS (Therapy Intelligent Personal Sensor). TIPS can capture contextual information (posture, physical activity, geoposition) and physiological information (heart rate, skin conductance, breath frequency). TIPS uses geoposition in order to register the nature, time and location of activities that the children carry out. With this system, the clinician has real access to patient's mobility habits.

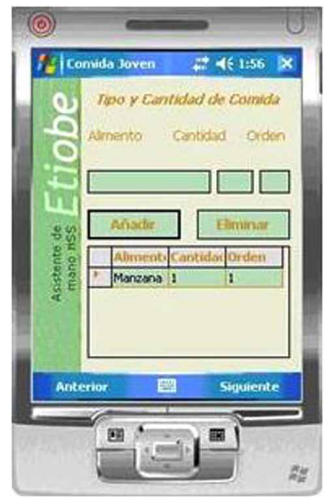

Fig. (8). A PDA food self-report register from the MSS.

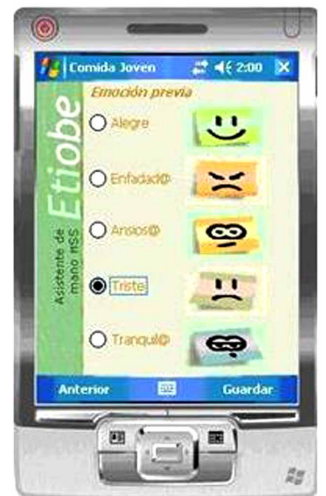

Fig. (9). A Self-report emotion diary from the MSS.

\section{CONCLUSION}

ETIOBE is an e-health system designed for the treatment of childhood weight loss. The real-time multi-modal nature of the ETIOBE programme provides a unique blend of qualities which lends itself to addressing the inherent difficulties incurred in the treatment of obesity in children. Obese children are surrounded by stimuli that drive them towards eating hypercaloric food and a sedentary lifestyle. For that reason, therapists need to be closely involved in the 'real' daily lives of the children they are treating in order to help change their eating and mobility habits.

Furthermore, another benefit of the ETIOBE system is the use of new technologies. Most children are "technological natives" and are completely accustomed to using technologies for both communication and entertainment. It is therefore anticipated that the familiarity of these media and new technologies like the Internet will enable children to feel comfortable with learning in this format. Another interesting aspect of the ETIOBE platform is that it allows for flexibility in adjusting treatment goals according to the progress of individual patients. Traditionally, homework tasks or weekly treatment goals (e.g. to go for a walk 20 minutes twice per week, or to have breakfast everyday) are set during face-toface clinical appointments, and they cannot be checked until the next session. In ETIOBE the completion of tasks can be checked in real time, which can then be used to shape reinforcements, and minimise the risk of failure due to children losing interest or feeling disheartened with the program.

A growing proportion of children in western countries are in need of more effective therapeutic management to treat or reduce the risk of obesity. This problem is overwhelming the capacities of the European public health systems, meaning that many children do not receive effective treatments or sufficient preventative tools. Given the current lack of evidence-based treatments for childhood obesity, governments are now recognising that there is an urgent need to prioritise and support the development of suitable and effective treatments in this area [1]. ITCs, such as the ETIOBE system have unique properties which enhance motivation, engagement and treatment adherence by capitalising on childrens' familiarity with technology and adapting treatment goals in alignment with real-time monitoring of progress.

\section{ACKNOWLEDGEMENT}

Funding for the study was provided by grants PSI200804392/PSIC from de MICINN (Spain), PROMETEO/2008/ 157, and CIBER Fisiopatologia de la Obesidad y Nutricion ISCIII CB06/03/0052 from the Spanish Government.

\section{REFERENCES}

[1] Obesity in Europe: The case for Action [monograph on Internet] European Association for the Study of Obesity. Available from: www.iotf.org.

[2] Guo SS, Wu W, Chumlea WC, Roche AF. Predicting overweight and obesity in adulthood from body mass index values in childhood and adolescence. Am J Clin Nutr 2002; 76: 653-8.

[3] National Institute for Health and Clinical Excellence. Obesity: Guidance on the prevention, identification, assessment and management of overweight and obesity in adults and children. Available from http://www.nice.org.uk/guidance/CG43

[4] National Health and Medical Research Council from Australia. Clinical Practice Guidelines for the Management of Overweight 
and obesity in Adults. Available from www.obesityguidelines.gov.au

[5] Gilles A, Cassano M, Shepherd EJ, Higgins D, Hecker JE, Nangle DW. Comparing active pediatric obesity treatments: using metaanalysis. J Clin Child Adolesc 2008; 37: 886-92.

[6] Sarwer DB, Foster GD, Wadden TA. Treatment of obesity I: Adult obesity. Thompson EJK, Ed. Handbook of eating disorders and obesity. New York. Wiley and Sons. 2004.

[7] National Heart, Lung, and Blood Institute. The practical guide: identification, evaluation, and treatment of overweight and obesity in adults. Bethesda, National Institutes of Health, NIH publication 00-4084. Available at www.nhlbi.nih.gov/ guidelines/obesity/ob_home.htm

[8] Wadden TA, Stenberg JA, Letizia KA, Stunkard AJ, Foster GD. Treatment of obesity by very-low-calorie diet, behaviour therapy and their combination: A five-year perspective. Int J Obes 1990; 51: 167-72.

[9] Elfhag K, Rossner S. Who succeeds in maintaining weight loss? A conceptual review of factors associated with weight loss maintenance and weight regain. Obes Rev 2005; 6: 67-85.

[10] Wilson GT, Brownell KD. Behavioral treatment for obesity. In Fairburn CG. Brownell, KD (Eds). Eating Disorders and Obesity: A Comprehensive Handbook. The Guilford Press: New York 2003; pp 524-8.
[11] Garris R, Ahlers R, Driskell J. Games, motivation and learning: a research and practice model. Simulation Gaming 2002; 33: 441-67.

[12] Streit KJ, Stevens NH, Stevens V, Rossner J. Food records: A predictor and modification of weight change in a long-term weight loss program. J Am Diet Assoc 1991; 91: 13-216.

[13] Stone AA, Shiffman S, Schwartz JE, Broderick JE, Hufford MR. Patient non-compliance with paper diaries. Br Med J 2002; 324 1193-4.

[14] Yon BA, Johnson RK, Harvey-Berino J, Gold BC, Howard AB. Personal digital assistants are comparable to traditional dietary selfmonitoring during a weight loss program. J Behav Med 2007; 30: 165-75.

[15] Lee NJ, Bakken S. Development of a prototype personal digital assistant-decision support system for the management of adult obesity. Int J Med Inform 2007; 76: 281-92.

[16] Beasley M, Riley WT, Davis A, Singh J. Evaluation of a PDAbased dietary assessment and intervention program: a randomized controlled trial. J Am Coll Nutr 2008; 27: 380-6.

[17] Fowles ER, Gentri B. The feasibility of Personal Digital Assistants (PDAs) to collect dietary intake data in low-income pregnant women. J Nutr Educ Behav 2008; 40: 374-7.

[18] Baños R, Cebolla A, Zaragoza I, Botella C, Alcañiz M. Electronic PDA dietary and physical activity registers in a weight loss tretment program for children: a description of the ETIOBE personal digital assistant system. J Cyber Rehabil 2009; 2: 235-41.

Received: June 27, 2010

Revised: July 28, 2010

Accepted: August 24, 2010

(C) Baños et al.; Licensee Bentham Open.

This is an open access article licensed under the terms of the Creative Commons Attribution Non-Commercial License (http://creativecommons.org/licenses/by-nc/3.0/) which permits unrestricted, non-commercial use, distribution and reproduction in any medium, provided the work is properly cited. 